\title{
Evaluation of the HER2 and Hormone Receptor Status in Metastatic Breast Cancer Using Cell Blocks: A Multi-Institutional Study
}

\author{
Rieko Nishimura $^{a}{ }^{f} \quad$ Yuya Murata $^{b}$ Kiyoshi Moric Katsushige Yamashiro ${ }^{d}$ \\ Kazuya Kuraoka ${ }^{e}$ Shu Ichihara $^{f}$ Kenichi Taguchi $^{g}$ Hiroyoshi Suzuki $^{\text {h }}$ \\ Masahiro Ito ${ }^{i}$ Natsumi Yamashita ${ }^{j}$ \\ ${ }^{a}$ Department of Clinical Laboratory, Shikoku Cancer Center, Matsuyama, Japan; ${ }^{b}$ Department of Pathology, Tokyo \\ Medical Center, Tokyo, Japan; ' Department of Pathology, Osaka National Hospital, Osaka, Japan; ${ }^{\mathrm{d}}$ Division of \\ Pathology, Hokkaido Cancer Center, Sapporo, Japan; ${ }^{e}$ Department of Diagnostic Pathology, Kure Medical Center \\ and Chugoku Cancer Center, Kure, Japan; ' Department of Pathology, Nagoya Medical Center, Nagoya, Japan;

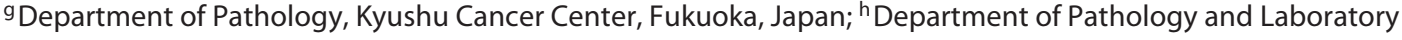 \\ Medicine, Sendai Medical Center, Sendai, Japan; 'Clinical Research Center, Nagasaki Medical Center, Omura, Japan; \\ jDivision of Clinical Biostatistics, Section of Cancer Prevention and Epidemiology, Clinical Research Center, Shikoku \\ Cancer Center, Matsuyama, Japan
}

\section{Keywords}

Cytology $\cdot$ Breast cancer metastases $\cdot$ Hormone receptors · HER2 Cell block

\begin{abstract}
Objective: We explore the problems associated with the cell block (CB) method for receptor analysis in breast cancer metastases and propose a method for reporting the results. Study Design: Nine institutions used the CB method for the analysis of hormone receptors (HRs) and HER2 (human epidermal growth factor receptor 2) protein in cytological specimens of breast cancer metastases in routine practice. The stained slides were independently evaluated by 8 pathologists. Dual in situ hybridization assay was performed in cases
\end{abstract}

\begin{tabular}{ll}
\hline KARGER & (c) 2018 The Author(s) \\
Published by S. Karger AG, Basel & Karger \\
E-Mail karger@karger.com & This article is licensed under the Creative Commons Attribution- \\
www.karger.com/acy & $\begin{array}{l}\text { NonCommercial-NoDerivatives 4.0 International License (CC BY- } \\
\text { NC-ND) (http://www.karger.com/Services/OpenAccessLicense). } \\
\text { Usage and distribution for commercial purposes as well as any dis- } \\
\text { tribution of modified material requires written permission. }\end{array}$
\end{tabular}

of discordant results for HER2 protein. Based on the results, we propose a method for receptor scoring in the CB method. Results: Of 61 specimens, 57 contained tumor cells. Two or more pathologists disagreed on the results for the estrogen receptor, progesterone receptor, and HER2 protein in 3 (5.3\%), $13(22.8 \%)$, and 19 (33.3\%) cases, respectively. The discrepant results for the HRs were attributed to the presence of a few positive cells or faintly stained cells. The high interobserver discordance rate for HER2 protein was explained by interobserver differences in the scoring criteria. Conclusion: The use of categorical scoring into positive and negative is recommended for evaluating the HR expressions. Use of strict criteria for HER2 protein 2+ and 3+ cases is recommended, as HER2-positive cases should not be missed.

(C) 2018 The Author(s)

Published by S. Karger AG, Basel

Correspondence to: Rieko Nishimura

Department of Pathology, National Hospital Organization, Nagoya Medical Center 4-1-1 Sannomaru

Naka-ku, Nagoya, Aichi 460-0001 (Japan)

E-Mail rnishimura-path@ umin.ac.jp 


\section{Introduction}

The American Society of Clinical Oncology/College of American Pathologists (ASCO/CAP) guidelines [1, 2] recommend receptor analysis for both hormone receptors (HRs) and HER2 (human epidermal growth factor receptor 2) in cytologic samples obtained from sites of disease recurrence or metastasis in breast cancer patients, because the receptor status may differ between the primary tumor and recurrent tumor. Cytological analysis can be applied to several types of metastatic lesions, as well as to body cavity fluids, and is useful for patients in a poor general condition. Therefore, use of cytology specimens for receptor analysis is a rapid, inexpensive, and less invasive alternative to biopsy at metastatic sites and in metastatic breast cancer patients.

Some studies have been reported in which the results of analysis for receptors in fine-needle aspiration cell blocks (CBs) fixed in 10\% buffered formalin showed excellent agreement with those in the corresponding tissue blocks, including for HRs at primary sites [3] or solid tumors at metastatic sites [4], and for HER2 at the primary sites $[5,6]$. However, in the case of receptor analysis in cytological specimens prepared from body cavity fluids, the method of preparation of the specimens and of receptor scoring in the stained slides has not yet been thoroughly studied. In addition, there are no reports of comparison of the use of the above-mentioned methods in routine clinical practice among institutions.

The first author and her colleagues at the Shikoku Cancer Center recommend the use of the $\mathrm{CB}$ method as a suitable method for receptor analysis in cytological specimens [5, 7], and have been routinely using this method for receptor analysis at their institution since August 2011. Herein, we report the results of a multi-institutional study, wherein receptor analysis was performed in cytological specimens obtained from breast cancer metastatic sites. The CB method used at the Shikoku Cancer Center was used at each of the institutions. The interobserver reproducibility of staining of the sections was evaluated. We discuss the problems associated with this procedure and propose an appropriate way to report the results.

\section{Materials and Methods}

The analyses of the HRs and HER2 in the cytological specimens obtained from breast metastatic sites were conducted at 9 institutions affiliated to the National Hospital Organization (NHO): Hokkaido Cancer Center, Sendai Medical Center, Tokyo Medical
Table 1. Autostainers used at the institutions

\begin{tabular}{lll}
\hline Autostainers & \multicolumn{2}{l}{ Receptors (institutions), $n$} \\
\cline { 2 - 3 } & hormone receptors & HER2 protein \\
\hline Ventana Bench Mark (Roche) & 7 & 8 \\
Dako Autostainer (Agilent) & 1 & 1 \\
BOND (Leica) & 1 & 0 \\
\hline
\end{tabular}

HER2, human epidermal growth factor receptor 2.

Center, Nagoya Medical Center, Osaka National Hospital, Kure Medical Center and Chugoku Cancer Center, Nagasaki Medical Center, Kyushu Cancer Center, and Shikoku Cancer Center. The time frame for case registration was from April 1, 2015, to March 31,2016 . The receptor analysis was conducted in cytological specimens collected from breast cancer metastatic sites in patients who needed receptor analysis in routine practice. We prepared stained slides from patients who agreed, via signing an informed consent form, to have their slides sent outside the hospital.

The slides stained for evaluation of the HR and HER2 protein status were collected and independently evaluated by 8 pathologists. Then, the discordance rates among the observers were calculated, and the interobserver agreement level was also quantitated. HER2 dual in situ hybridization (DISH) assay was conducted in all the cases with discordant results for HER2 protein expression. The types of discordant cases and the reasons for the discordance were discussed.

\section{Cell Block Preparation}

All of the participant institutions used the same method to prepare the CBs. The specimens collected from the metastatic sites were fixed in $10 \%$ buffered formalin for $6-48 \mathrm{~h}$, processed for CB preparation by the sodium alginate method, and embedded in paraffin. In brief, the sample-containing tube was centrifuged at 3,000 rpm for $5 \mathrm{~min}$, formalin was removed, $0.5 \mathrm{~mL}$ of $1 \%$ sodium alginate was added, the tube was centrifuged again at 3,000 rpm for $5 \mathrm{~min}$, and $0.5 \mathrm{~mL}$ of $1 \mathrm{M}$ calcium chloride was added. The gel pellet formed by this process was embedded in paraffin, and the paraffin $\mathrm{CB}$ was prepared in the same way as for histological specimens.

\section{Staining for the Receptors}

Sections prepared from the CBs were stained with HE and for immunohistochemistry (IHC). The autostainers at each institution were used for the IHC staining (Table 1). The Ventana BenchMark (Roche Diagnostics, Basel, Switzerland) and DAKO Autostainer (Agilent, Santa Clara, CA, USA) were used for both the HR and HER2 protein stainings in 7 and 1 institution, respectively. At 1 of the institutions, BOND (Leica Biosystems, Nussloch, Germany) was used for the HR staining and Ventana BenchMark for the HER2 protein staining. The HER2 DISH assay was conducted using Ventana BenchMark at the Shikoku Cancer Center.

\section{Evaluation of HR Expression}

Staining for the HRs was rated as positive or negative according to the following criteria: any nuclear staining of tumor cells was considered as positive, and an absence of nuclear staining was con-
2
Acta Cytologica

DOI: $10.1159 / 000488382$
Nishimura et al. 
sidered as negative. The percentage of stained tumor cells was not considered for the evaluation because it is difficult to estimate the percentage of stained cells among the tumor cells in cytological specimens, which also contain several nonneoplastic cells.

\section{Evaluation of HER2 Protein Expression}

The staining results for HER2 protein were scored as $0,1+, 2+$, or $3+$, according to the following criteria: $3+$, strong circumferential membranous staining of the tumor cells; $2+$, moderate circumferential staining of the tumor cells; $1+$, weak and incomplete membranous staining of the tumor cells; 0 , absent staining. Again, the percentage of stained tumor cells was not considered for scoring because it is difficult to estimate the percentage of stained cells among the tumor cells in cytological specimens, which also contain several nonneoplastic cells. The HER2 protein expression status was categorized as negative in slides assigned a score of 0 or $1+$, as equivocal result in slides assigned a score of $2+$, and as positive in slides assigned a score of $3+$.

\section{Evaluation of the Results of the HER2 DISH Assay}

The INFORM HER2/neu Dual ISH DNA Probe Cocktail assay was used on the sections prepared from the CBs. The DISH assay was performed according to the manufacturer's recommended protocol for surgical specimens. The HER2/neu (black) to chromosome enumeration probe 17 (CEP17; red) ratio was manually counted under a light microscope in each specimen by 2 investigators to avoid subjective bias. At least 20 cells were counted.

The criteria consisted of a combination of the HER2/CEP17 ratio and average number of HER2 signals per cell. The result for HER2 gene expression was "amplified," if the HER2/CEP17 signal count ratio was 2.0 or if the signal count ratio was $<2.0$, but the average number of HER2 signals per cell was 6.0, "equivocal" if the HER2/CEP17 signal count ratio was $<2.0$ and the average number of HER 2 signals per cell was 4.0 but $<6.0$, and "not amplified" if the HER2/CEP17 signal count ratio was $<2.0$ and the average number of HER2 signals was $<4.0$.

\section{Data Management}

The degree of interobserver agreement was quantitated using the generalized kappa and pairwise kappa statistics. The pairwise kappa statistics were used to calculate the proportion of cases in which 2 observers agreed, adjusted for the level of agreement that would be expected to occur solely by chance. The generalized kappa represented a summary of the agreement across all observers. The Fleiss-Cohen-weighted kappa coefficient was used to assess the HER2 protein staining results. The correlation was scored as "moderate" if the kappa value exceeded 0.4, "good" if it exceeded 0.6 , and as "excellent" if it exceeded 0.8. The weighted kappa coefficient was calculated using Microsoft Office Excel 2016 software.

\section{Results}

\section{Registered Specimens}

A total of 62 specimens were registered, of which 61 were reviewed. The types of specimens were as follows: pleural fluid (44 specimens), ascitic fluid (14 specimens), lymph node ( 2 specimens), pericardial fluid (1 specimen),
Table 2. Interobserver discordance in the 57 cases reviewed

\begin{tabular}{llc}
\hline Receptors & \multicolumn{2}{l}{ Discordant cases, $n(\%)$} \\
\cline { 2 - 3 } & all cases & $\begin{array}{l}\text { discordant between } 2 \\
\text { or more observers }\end{array}$ \\
\hline ER & $11(19.3)$ & $3(5.3)$ \\
PR & $18(31.6)$ & $13(22.8)$ \\
HER2 & $25(43.8)$ & $19(33.3)$ \\
\hline
\end{tabular}

ER, estrogen receptor; PR, progesterone receptor; HER2, human epidermal growth factor receptor 2 .

and a subcutaneous metastatic nodule on the back of a patient ( 1 specimen). Four of the 62 specimens (6.5\%) did not contain tumor cells, which included 2 specimens each of pleural fluid and ascitic fluid.

\section{Number of Specimens Scored by the Pathologists and} the Backgrounds of the Pathologists

Sixty-one out of the 62 specimens were reviewed because 1 specimen could not be gathered. Of the 61, 57 specimens contained tumor cells. Five of the 8 pathologists evaluated all of the 57 specimens containing tumor cells, 1 pathologist evaluated 55 specimens, and 2 pathologists evaluated 48 specimens.

The 8 pathologists had an average experience of 20 years each (range 15-36) in the field of surgical pathology. The pathologists were between 41 and 64 years old (average age 51 years).

\section{Interobserver Discordance in the Staining Results for} the HRs

For the case of the estrogen receptor (ER), there were disagreements in $11(19.3 \%)$ out of the 57 cases. In 8 of these cases only 1 pathologist disagreed with the verdict of the remaining pathologists, while there were broader disagreements in the remaining 3 cases (5.3\%; Table 2). The generalized kappa value indicated good agreement (0.777). The pairwise kappa values for agreement among the observers ranged from moderate to excellent (0.5440.948; Table 3).

For the case of the progesterone receptor (PR), there were disagreements in $18(31.6 \%)$ out of the 57 cases. In 5 of 18 cases, only 1 pathologist disagreed with the verdict of the remaining pathologists, while there were broader disagreements in the remaining 13 cases (22.8\%; Table 2). The generalized kappa value indicated good agreement (0.695). The pairwise kappa values for agreement among
Evaluation of HER2 and HR Status in Metastatic Breast Cancer Using CBs 
Table 3. Pairwise interobserver agreement for the results of ER staining according to the kappa statistic

\begin{tabular}{|c|c|c|c|c|c|c|c|}
\hline \multirow{2}{*}{$\begin{array}{l}\text { Ob- } \\
\text { server }\end{array}$} & \multicolumn{7}{|c|}{ Observer } \\
\hline & 2 & 3 & 4 & 5 & 6 & 7 & 8 \\
\hline 1 & 0.864 & 0.773 & 0.582 & 0.834 & 0.787 & 0.888 & 0.886 \\
\hline 2 & & 0.751 & 0.604 & 0.749 & 0.769 & 0.813 & 0.806 \\
\hline 3 & & & 0.544 & 0.740 & 0.800 & 0.788 & 0.836 \\
\hline 4 & & & & 0.695 & 0.731 & 0.748 & 0.544 \\
\hline 5 & & & & & 0.852 & 0.947 & 0.786 \\
\hline 6 & & & & & & 0.948 & 0.846 \\
\hline 7 & & & & & & & 0.837 \\
\hline
\end{tabular}

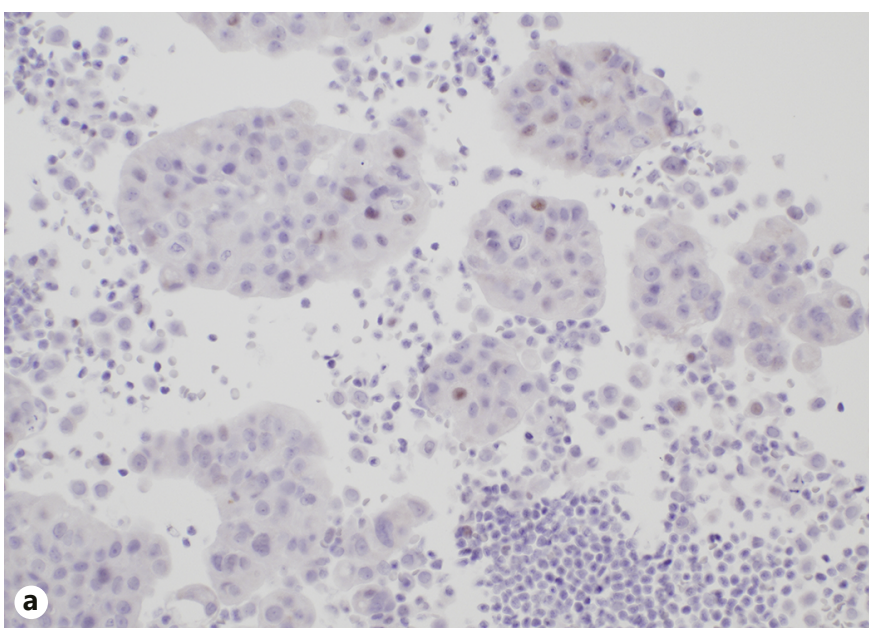

Table 4. Pairwise interobserver agreement for the results of PR staining according to the kappa statistic

\begin{tabular}{|c|c|c|c|c|c|c|c|}
\hline \multirow{2}{*}{$\begin{array}{l}\text { Ob- } \\
\text { server }\end{array}$} & \multicolumn{7}{|c|}{ Observer } \\
\hline & 2 & 3 & 4 & 5 & 6 & 7 & 8 \\
\hline 1 & 0.804 & 0.844 & 0.565 & 0.594 & 0.713 & 0.739 & 0.854 \\
\hline 2 & & 0.680 & 0.543 & 0.595 & 0.690 & 0.772 & 0.770 \\
\hline 3 & & & 0.491 & 0.554 & 0.669 & 0.688 & 0.846 \\
\hline 4 & & & & 0.747 & 0.806 & 0.695 & 0.504 \\
\hline 5 & & & & & 0.725 & 0.761 & 0.536 \\
\hline 6 & & & & & & 0.908 & 0.652 \\
\hline 7 & & & & & & & 0.704 \\
\hline
\end{tabular}

Fig. 1. Examples of discrepant results for HRs. A case of discordant results for the ER with some faintly stained cells (a) and a case of discordant results for the PR with a few positive cells (b). Some observers categorized the sections as negative.
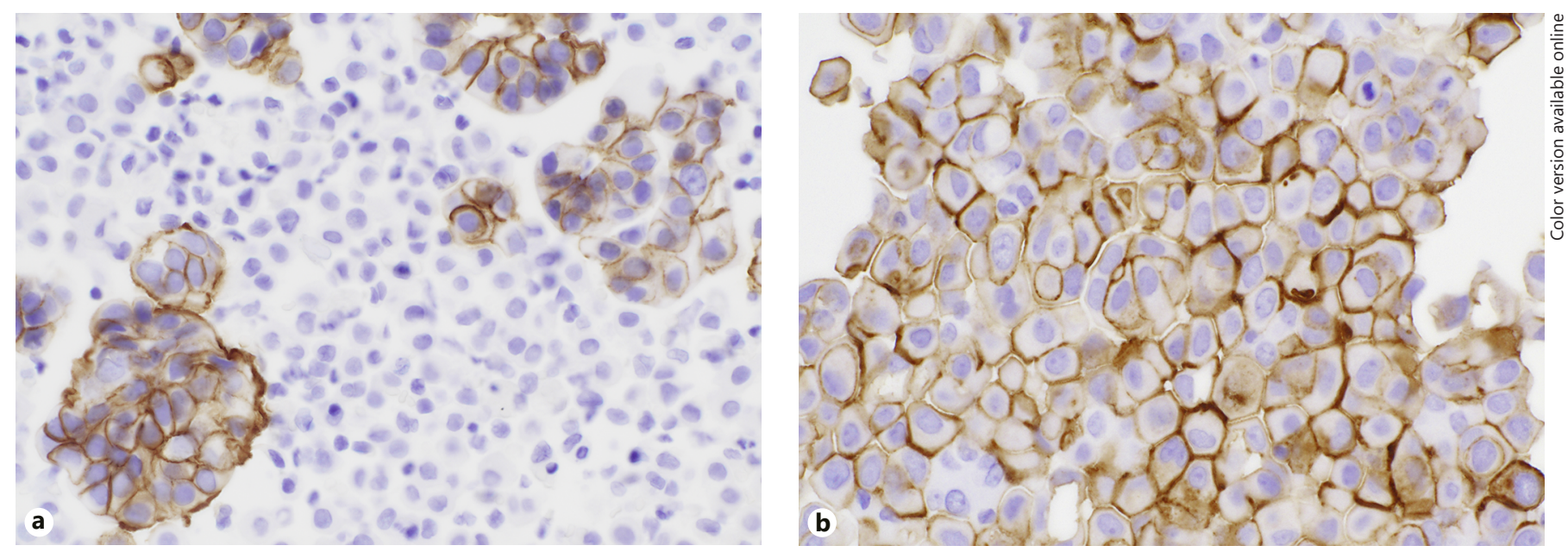

Fig. 2. Examples of disagreement between equivocal and positive results for HER2 protein. The results of the DISH assay were "amplified" (a) and "unamplified" (b). 
Table 5. Cases with discordant results of HER2 protein staining between 2 or more pathologists

\begin{tabular}{lcllll}
\hline HER2 protein staining results & Cases, $n$ & \multicolumn{2}{l}{ DISH assay results } \\
\cline { 3 - 5 } & & negative & equivocal & positive \\
\hline Negative/equivocal & 7 & 6 & 1 & 0 \\
Equivocal/positive & 5 & 2 & 0 & 3 \\
Negative/equivocal/positive & 7 & 7 & 0 & 0 \\
\hline Total & 19 & 15 & 1 & 3 \\
\hline
\end{tabular}

HER2, human epidermal growth factor receptor 2; DISH, dual in situ hybridization. observers ranged from moderate to excellent (0.4910.908; Table 4). The discrepancies in the results for the HRs were considered to be attributable to the presence of a few positive cells or faintly stained cells (Fig. 1).

\section{Interobserver Discordance in the Staining Results for HER2}

The 3-level categorization of negative, equivocal, and positive was used to assess the interobserver disagreements. There were disagreements in $25(43.8 \%)$ out of the 57 cases. In 6 of the 25 cases only 1 pathologist disagreed with the verdict of the remaining pathologists, while there were broader disagreements in the remaining 19 cases (33.3\%; Table 2). Among these 19 cases, the disagreement was between negative and equivocal in 7 cases, between equivocal and positive in 5 cases, and among all 3 categories in 7 cases (Table 5). The generalized kappa value indicated good agreement (0.635). The pairwise kappa values for agreement among observers ranged from poor to excellent (0.336-0.816; Table 6).

DISH assay was performed in the 25 cases in which the results of IHC were discordant. There were 3 cases that were labeled as "amplified" by the DISH assay. In all 3 cases the disagreement was between equivocal and positive by IHC. The DISH results of the 19 cases in which there was disagreement in the results of IHC between 2 or more pathologists are summarized in Table 5. Among the 7 cases where the disagreement was between negative and equivocal results of IHC, the DISH result was "unamplified" in 6 cases and "equivocal" in 1 case. Among the 5 cases with disagreement between equivocal and positive results of IHC, the result of the DISH assay was "unamplified" in 2 cases and "amplified" in 3 cases (Fig. 2).

The results of the DISH assay were "unamplified" in all 7 cases that were classified into all 3 different categories by different pathologists (Table 5). The IHC result in 6 of these 7 cases was classified as negative by the criteria of
Table 6. Pairwise interobserver agreement for the results of HER2 protein staining according to the kappa statistic

\begin{tabular}{|c|c|c|c|c|c|c|c|}
\hline \multirow{2}{*}{$\begin{array}{l}\text { Ob- } \\
\text { server }\end{array}$} & \multicolumn{7}{|c|}{ Observer } \\
\hline & 2 & 3 & 4 & 5 & 6 & 7 & 8 \\
\hline 1 & 0.593 & 0.597 & 0.669 & 0.798 & 0.813 & 0.816 & 0.789 \\
\hline 2 & & 0.336 & 0.487 & 0.749 & 0.606 & 0.411 & 0.561 \\
\hline 3 & & & 0.663 & 0.436 & 0.492 & 0.614 & 0.718 \\
\hline 4 & & & & 0.493 & 0.696 & 0.594 & 0.757 \\
\hline 5 & & & & & 0.665 & 0.710 & 0.603 \\
\hline 6 & & & & & & 0.738 & 0.716 \\
\hline 7 & & & & & & & 0.674 \\
\hline
\end{tabular}

HER2, human epidermal growth factor receptor 2.

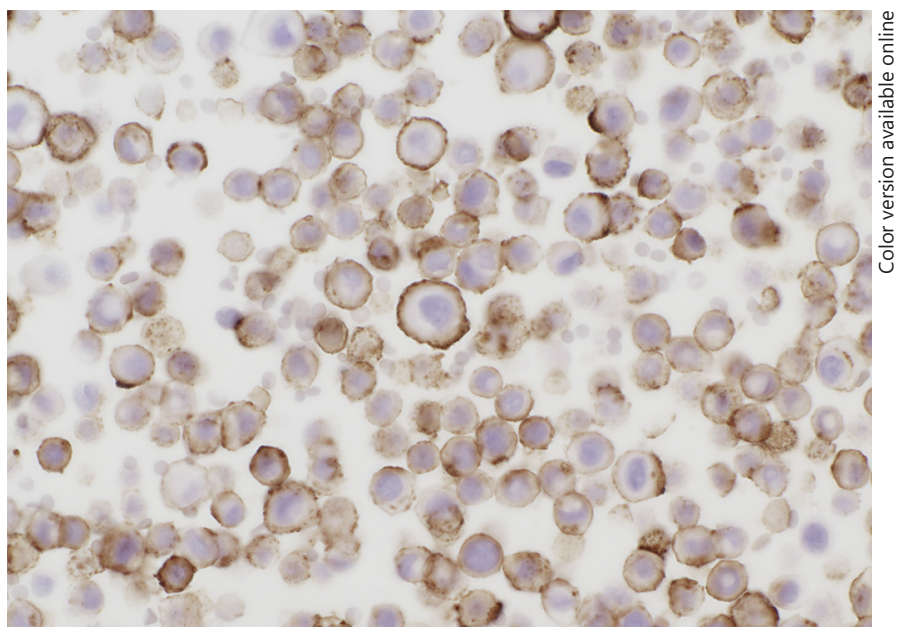

Fig. 3. A case in which the results of HER2 protein staining were interpreted as being positive, negative, or equivocal (all different categories) by different observers. This case was categorized as positive by 2 pathologists, as equivocal by 4 pathologists, including the first author, and as negative by 1 pathologist. The result of the DISH assay in this case was "unamplified."
Evaluation of HER2 and HR Status in Metastatic Breast Cancer Using CBs 
the first author (R.N.), and in the remaining case was classified as equivocal by the same author (Fig. 3). Four cases were classified as negative or equivocal by all but 1 pathologist and 1 case was classified as equivocal or positive by all but 1 pathologist. One case was classified as negative by 5 pathologists, but as equivocal and positive by 2 other pathologists. One case was classified as negative by 2 pathologists, as equivocal by 4 pathologists, and as positive by 3 pathologists. The DISH result was "unamplified" in all of these cases.

\section{Discussion}

This is the first report of receptor analysis in cytological specimens obtained from breast metastatic sites conducted systematically at different institutions. The receptor analysis in sections prepared from CBs was easy to perform at these institutions, where receptor analysis in histological specimens is routinely conducted. The most frequently obtained specimen type was pleural fluid (44 specimens) followed by ascitic fluid (14 specimens). This method can also be applied in patients with allergy to local anesthetic drugs

Three types of autostainers were used in our study (Table 1). The staining protocol employed was the same as that used for histological sections. There were no differences in the interobserver discordance rates among the 3 types of autostainers.

The interobserver discordance rates for HRs and HER2 protein in the specimens prepared from the CBs were higher than those in the sections prepared from histological specimens [8-11]. This is considered to be because of a worse condition of cytological specimens as compared to tissue specimens. Some cytological specimens contain numerous inflammatory cells, making it sometimes difficult to identify tumor cells in cytological sections, especially in receptor-negative cases. The use of immunostains specific to breast cancer cells, e.g., antibodies against GATA3 or gross cystic disease fluid protein-15 (GCDFP-15), is recommended for easier detection of the tumor cells in these cases. In addition, the cells in body cavity fluids may sometimes become degenerative if there is some delay before fixation. Therefore, it is important to fix the cells as soon as possible after the samples are obtained. We also recommend that the gel pellets containing the cells be placed sagittally in the cassette before they are embedded in paraffin, so that all the cell layers can be seen and it becomes easier to detect the tumor cells on the slide. For the case of HER2 protein, the in- terobserver differences in the scoring criteria influenced the high interobserver discordance rate.

The same standard receptor assessment criteria used in histological sections should also be used, as much as possible, for cytological specimens. However, we did not include the percentage of positive cells in our assessment because it was difficult to estimate the percentage of positively stained cells in several cytological specimens in our study. Twenty of the 57 (35.1\%) specimens contained many nonneoplastic cells in the background. Especially in the specimens of body cavity fluids, the tumor cells were mixed with inflammatory cells and mesothelial cells, so that the precise percentage of tumor cells could not be assessed.

Fluorescence in situ hybridization (FISH) assay is the standard method to detect HER2 gene amplification in tissue sections. However, it is difficult to distinguish scattered tumor cells among the many nonneoplastic cells under the dark-field fluorescence microscope. Thus, we believe that bright-field HER2 DISH assay is a better choice of method than FISH assay to assess the HER2 gene amplification status in cytological specimens in routine clinical practice, especially of body cavity fluids obtained from metastatic sites.

Based on our study results, we propose an appropriate method to report the results of the receptor status in sections prepared from CBs. For evaluating the results of HR staining, we recommend reporting the results as positive or negative according to the following criteria: any nuclear staining of tumor cells should be classified as positive, and a complete lack of staining of tumor cells as negative. For HER2, the same ASCO/CAP criteria as those used for histological sections [2] can be used for the sections prepared from CBs. HER2 protein 2+ cases should be examined by DISH assay. It is important to use strict scoring criteria to categorize specimens as $2+$ or $3+$, so as not to miss the HER2-positive cases. Only strongly stained cells should be categorized as $3+$, and cases where it is difficult to decide between $2+$ and $1+$ should be labeled as $2+$.

To validate the recommended criteria for evaluating the HR and HER2 statuses using CBs, the slides were evaluated again by the same 8 pathologists according to the criteria. In this evaluation of the HR staining status, the number of cases of disagreement on the results between 2 or more pathologists was reduced (ER, 2 cases, 3.5\%; PR, 9 cases, 15.8\%) compared to the first evaluation, although the interobserver discordance rate (which included cases where only 1 pathologist reported a discordant result) was almost the same for ER (10 cases, 17.5\%) and higher for PR (26 cases, $45.6 \%$ ). The high discordance rate 
for $\mathrm{PR}$ is explained by the fact that 3 pathologists tended to rate as negative those cases in which there was only a small number of faintly stained tumor cells. According to this result, the problem in HR staining evaluation is a criterion for the faintly stained cells. Meanwhile, the number of disagreements in relation to the HER2 protein status increased to 40 (70.2\%). In 37 of the 40 cases, there were disagreements in the results of evaluation of the HER2 status, including between intermediate and negative results in 29 cases, and between intermediate and positive results in 8 cases. However, the number of cases with disagreements among all 3 categories decreased, although they were not entirely eliminated ( 3 cases). According to this result, adoption of the recommended criteria may be rational because of the decrease in the number of cases of disagreements in all 3 categories. However, it would be difficult to say the same with regard to evaluation of the HER2 protein status. Based on our findings, HER2 protein $2+$ cases should be examined by DISH assay.

In conclusion, we conducted a multi-institutional study of receptor analysis in cytological specimens obtained from sites of metastasis from breast cancer. For obtaining specimens for staining, we recommend the preparation of CBs fixed in $10 \%$ buffered formalin for 6-48 $\mathrm{h}$ followed by the sodium alginate method. Use of categorical scoring into positive and negative is recommended for evaluating the HR expressions. For HER2 protein evaluation, it is important to use strict scoring criteria for $2+$ or $3+$, and HER 2 protein $2+$ cases should be examined by DISH assay.

\section{Acknowledgements}

This study was supported by a Grant-in Aid for Clinical Research from the National Hospital Organization (H26-NHO Gan Ippan-02 and H29-NHO Gan Ippan-02). We thank the other members of the research group sponsored by the grant.

\section{Statement of Ethics}

The study plan was approved by the central ethical committee of the National Hospital Organization on January 9, 2015.

\section{Disclosure Statement}

The authors have no conflicts of interests to declare.

\section{References}

1 Hammond MEH, Hayes DF, Dowsett M, Allred DC, Hagerty KL, Badve S, Fitzgibbons PL, Francis G: American Society of Clinical Oncology/College of American Pathologists guideline recommendations for immunohistochemical testing of estrogen and progesterone receptors in breast cancer. J Clin Oncol 2010;28:2784-2795.

-2 Wolff AC, Hammond MEH, Hicks DG, Dowsett M, McShane LM, Allison KH, Allred DC, Bartlett JMS, Bilous M, Fitzgibbons P, Hanna W, Jenkins RB, Mangu PB, Paik S, Perez EA, Press MF, Spears PA, Vance GH, Viale G, Hayes DF: Recommendations for human epidermal growth factor receptor 2 testing in breast cancer: American Society of Clinical Oncology/College of American Pathologists clinical practice guideline update J Clin Oncol 2013;31: 3997-4013.

-3 Kumar S K, Gupta N, Rajwanshi A, Joshi K, Singh G: Immunochemistry for oestrogen receptor, progesterone receptor and HER2 on cell blocks in primary breast carcinoma. Cytopathology 2012;23:181-186.
4 Monaco SE, Wu Y, Teot LA, Cai G: Assessment of estrogen receptor (ER), progesterone receptor (PR), and human epidermal growth factor receptor 2 (HER2) status in the fine needle aspirates of metastatic breast carcinomas. Diagn Cytopathol 2013;41:308-315.

-5 Nishimura R, Okamoto N, Satou M, Kojima K, Tanaka S: HER 2 immunohistochemistry for breast cancer cell blocks can be used in the same way as that used for histological specimens. Diagn Cytopathol 2016;44:274-279.

6 Vohra P, Buelow B, Chen YY, Serrano M, Vohra MS, Berry A, Ljung BM: Estrogen receptor, progesterone receptor, and human epidermal growth factor receptor 2 expression in breast cancer FNA cell blocks and paired histologic specimens: a large retrospective study. Cancer Cytopathol 2016;124: 828-835.

7 Nishimura R, Okamoto N, Satou M, Kojima K, Tanaka S, Yamashita N: Bright-field HER2 dual in situ hybridization (DISH) assay on breast cancer cell blocks: a comparative study with histological sections. Breast Cancer 2016;23:917-921.
$>8$ Hsu CY, Ho DM, Yang CF, Lai CR, Yu IT, Chiang $\mathrm{H}$ : Interobserver reproducibility of Her-2/neu protein overexpression in invasive breast carcinoma using the DAKO HercepTest. Am J Clin Pathol 2002;118:693-698.

-9 Tsuda H, Kurosumi M, Umemura S, Yamamoto S, Kobayashi T, Osamura RY: HER2 testing on core needle biopsy specimens from primary breast cancers: interobserver reproducibility and concordance with surgically resected specimens. BMC Cancer 2010;10:534.

10 Cohen DA, Dabbs DJ, Cooper KL, Amin M, Jones TE, Jones MW, Chivukula M, Trucco GA, Bhargava R: Interobserver agreement among pathologists for semiquantitative hormone receptor scoring in breast carcinoma. Am J Clin Pathol 2012;138:796-802.

11 Reisenbichler ES, Lester SC, Richardson AL, Dillon DA, Ly A, Brock JE: Interobserver concordance in implementing the $2010 \mathrm{ASCO} /$ CAP recommendations for reporting ER in breast carcinomas: a demonstration of the difficulties of consistently reporting low levels of ER expression by manual quantification. Am J Clin Pathol 2013;140:487-494.
Evaluation of HER2 and HR Status in Metastatic Breast Cancer Using CBs 